\title{
Effects of Combined Water Potential and Temperature Stresses on Cryptosporidium parvum Oocysts
}

\author{
MARK WALKER, ${ }^{*}$ KATHERINE LEDDY, AND ELAINE HAGAR \\ Natural Resources Department, University of Nevada, Reno, Nevada 89557-0013
}

\author{
Received 29 May 2001/Accepted 22 September 2001
}

\begin{abstract}
Hosts infected with the parasite Cryptosporidium parvum may excrete oocysts on soils in watersheds that supply public drinking water. Environmental stresses decrease the numbers of oocysts after deposition on soils. However, the rates and effects of combined stresses have not been well characterized, especially for the purposes of estimating decrease in numbers. We subjected oocysts to combined stresses of water potential $(-4$, -12 , and -33 bars), above-freezing temperatures $\left(4\right.$ and $\left.30^{\circ} \mathrm{C}\right)$, and a subfreezing temperature $\left(-14^{\circ} \mathrm{C}\right)$ for 1 , 14 , and 29 days and one to six freeze-thaw cycles $\left(-14\right.$ to $\left.10^{\circ} \mathrm{C}\right)$ to estimate coefficients to characterize population degradation using multiplicative error and exponential decay models. The experiments were carried out in $\mathrm{NaCl}$ solutions with water potentials of $-4,-12$, and -33 bars, in combination with temperature stresses at levels that could be expected in natural soils. Increased water potential increased the rate of population degradation for all temperature conditions investigated. Enhanced degradation leads to estimated rates of population degradation that are greater than those that have been reported and used in previous studies conducted to assess risk of water supply contamination from sources of $C$. parvum.
\end{abstract}

Large proportions of the oocysts of Cryptosporidium parvum shed on soils by infected hosts are probably destroyed by environmental stresses. Important stresses include temperature extremes, freeze-thaw cycling, and extreme water potential (especially desiccation) (20). It is unclear how predictably and at what rate degradation occurs when stresses are combined. Degradation rates are important to understand for the purpose of assessing the likelihood of contamination in the context of public drinking water supply protection. Models developed for the purpose of evaluating the risk of water contamination rely on estimates of coefficients that characterize decay rates before microbial contaminants are entrained and transported to surface waters (13). Risk assessments conducted to evaluate the contamination potential of sources of Cryptosporidium have relied on degradation rates from in situ studies (19). Firstorder decay approaches model Cryptosporidium degradation adequately, especially for conditions related to prolonged exposure to fixed temperatures (9). Present estimates of degradation coefficients are based on investigations of single stresses or uncontrolled combinations of several stresses $(9,16)$.

We tested the hypothesis that the interaction between temperature and water potential stresses enhances oocyst degradation, leading to rates of population decay that are higher than those previously reported and used for risk assessment. We evaluated this hypothesis using exponential decay models, with temperature and water potential stresses represented in a multiplicative error format (8) that related estimates of degradation coefficients to levels of stresses applied.

\section{MATERIALS AND METHODS}

Experimental design. We tested the effects of water potential, temperature, and freeze-thaw cycling on rates of oocyst degradation with a full-factorial

* Corresponding author. Mailing address: University of Nevada, Natural Resources Department (MS370-FA 132), 1664 Virginia Ave., Reno, NV 89557-0013. Phone: (775) 784-1938. Fax: (775) 784-4789. E-mail: mwalker@equinox.unr.edu. approach. Our design included three replicate trials at each combination of three lengths of exposure $\left(1,15\right.$, and 29 days), three temperatures $\left(-14,4\right.$, and $\left.30^{\circ} \mathrm{C}\right)$, and three levels of water potential $(-4,-12$, and -33 bars). We also examined the effects of freeze-thaw cycling at the three levels of water potential. We carried out 54 trials for above-freezing conditions (three replicates, two temperature levels, three durations of exposure, and three levels of water potential), 27 trials for below-freezing conditions (three replicates, three durations of exposure, and three levels of water potential), and 54 trials for freeze-thaw cycling (three replicates, three levels of water potential, and six freeze-thaw cycles).

Source of oocysts. Wild-type oocysts were extracted from feces obtained per rectum from naturally infected Holstein dairy calves in Fallon, Nevada. The purification procedure used successive differential sucrose gradients in a flowthrough centrifuge, as previously described for obtaining coccidial oocysts from poultry litter (18). Oocyst solutions were diluted with deionized, distilled water and stored at $4^{\circ} \mathrm{C}$ with $100 \mathrm{U}$ of penicillin $\mathrm{G}$ sodium, $100 \mu \mathrm{g}$ of streptomycin sulfate, and $0.25 \mu \mathrm{g}$ of amphotericin B per ml of oocyst suspension.

We conducted three analyses to verify that the Cryptosporidium species in the stock was most likely $C$. parvum. We examined samples of stock at a magnification of $\times 1,000$ by using differential interference contrast microscopy to verify that oocyst dimensions, geometry, and morphology corresponded with that expected of $C$. parvum oocysts (approximately spherical and 4 to $7 \mu \mathrm{m}$ in diameter [17]). We successfully applied fluorescein isothiocyanate-labeled, single-stage, anti-Cryptosporidium immunoglobulin M (Waterborne, Inc., New Orleans, La.). Finally, using PCR, we successfully amplified $C$. parvum DNA target sequences from stock solutions with forward and reverse primers provided by the National Institutes of Health AIDS Reagent Program (catalog no. 1558, forward primer, 5'-CCGAGTTTGATCCAAAAAGTTACGAAG-3'; and reverse primer, 5'-GC TCCTCATATGCCTTATTGAGTA-3'), using a previously described procedure (11).

We used stock diluted with distilled, deionized water containing approximately 50,000 oocysts/ml (estimated from 20 counts conducted on separate 100- $\mu$ l aliquots of stock). All experiments were carried out with stock solutions from the same batch, which was less than 2 months old before completion of all experiments. Immediately prior to carrying out trials, we characterized stock with respect to numbers present and proportions potentially effective, based on the criteria described below. Proportions considered to be potentially infective are denoted $v_{0}$.

Water potential solutions. One of the impediments to carrying out studies of the degradation of $C$. parvum oocysts in soils is poor recovery. Recovery efficiencies decrease significantly with time (for example, less than $2 \%$ is recovered 7 days after addition to soils $[10,12])$. It is unclear whether oocysts lost during soil sample processing represent a fraction that has been destroyed or whether they remain capable of infecting a new host in liquid or solid portions discarded during the extraction process. To avoid this uncertainty, we used osmotic poten- 
TABLE 1. Estimated first-order degradation coefficients ${ }^{a}$

\begin{tabular}{llcl}
\hline \multicolumn{1}{c}{ Temp } & \multicolumn{1}{c}{ Fitted model } & Model significance & Parameter significance \\
\hline 4 and $30^{\circ} \mathrm{C}$ & $k=0.003 \cdot$ bars $-0.004 \cdot$ Temp & $P<0.001$ & Temp, $P<0.001 ;$ Bars, $P=0.003$ \\
$-14^{\circ} \mathrm{C}$ & $k=0.013 \cdot$ bars & $P=0.001$ & Bars, $P=0.001$ \\
Freeze-thaw cycling $\left(-14\right.$ to $\left.10^{\circ} \mathrm{C}\right)$ & $k=0.055 \cdot$ bars & $P<0.001$ & Bars, $P<0.001$ \\
\hline
\end{tabular}

${ }^{a}$ Statistics are related to significance of regression models to estimate $k$ (degradation coefficient used to represent exponential rate of decay of oocyst populations: $\left.n_{3} / n_{1}=e^{\mathrm{kt}}\right)$ and coefficient estimates for temperature and water potential for above-freezing, subfreezing, and freeze-thaw cycling conditions, with $k=\ln \left(n_{3} / n_{1}\right) / t$ and $n_{3} / n_{1}$ representing the proportion of potentially infective oocysts remaining after $t$ days or $t$ freeze-thaw cycles.

tial as a surrogate for total water potential in soils. Stress on microbes due to water potential in soils is the result of the additive effects of matric and osmotic potentials (14). Previous work with fungi indicates that osmotic potential adequately simulates total water potential due to the combined effects of matric and osmotic potentials (1). Use of solutions avoids problems of uneven distribution of oocysts in soil, heterogeneity in soil moisture distribution, and inefficiencies in soil extraction methods. By the use of solutions, intact oocysts could be recovered by microfiltration.

We conducted preliminary trials with $\mathrm{NaCl}$ and sucrose solutions. We evaluated differences at three temperatures with respect to the relationship between estimates of the degradation coefficient (for $k$, the coefficient in the exponential model representing population change, see equation 1) and temperature and water potential. Preliminary results indicated that $\mathrm{NaCl}$ and sucrose solutions yielded equivalent results. For example, the results of trials conducted at 4, 25, and $30^{\circ} \mathrm{C}(n=41$ trials total $)$ at water potentials of $-6,-13,-20$, and -30 bars produced an estimate of $k=-0.002 \cdot$ bars $-0.003 \cdot$ temperature (regression significance, $P<0.001$; for coefficient significance, bars, $P=0.005$; and temperature, $P<0.001)$. These estimates are within the $95 \%$ confidence intervals for estimates derived from similar conditions in experiments carried out with $\mathrm{NaCl}$ (Table 1). Accordingly, we narrowed the scope of our experiments to $\mathrm{NaCl}$.

We prepared $0.08,0.27$, and 0.77 molal solutions of distilled, deionized water with $\mathrm{NaCl}$, which produced total water potential conditions of $-4,-12$, and -33 bars, as determined by thermocouple psychrometry (15). These levels simulate soil matric potentials that lie between field capacity ( -0.1 bars), the wilting point ( -15 bars), and extremely dry conditions (less than -15 bars) (2). The solutions of $0.08,0.27$, and $0.77 \mathrm{M} \mathrm{NaCl}$ had estimated freezing points of $-0.3,-0.8$, and $-2.9^{\circ} \mathrm{C}$, respectively, which were well above the minimum temperature $\left(-14^{\circ} \mathrm{C}\right)$ used for experiments.

Isolation of oocysts from suspensions. We isolated oocysts by vacuum-filtering an oocyst suspension through a $0.2-\mu \mathrm{m}$-pore-size diameter filter at a rate of approximately $1 \mathrm{ml}$ per min. The entire volume of each replicate, including approximately $1 \mathrm{ml}$ of distilled, deionized water used to rinse tubes after initial filtration, was filtered.

We tested recovery by comparing results of 10 replicate trials with direct counts of 20- $\mu$ l aliquots of stock solution. Results from the two methods were not significantly different (using the criterion of $\alpha=0.05$ ) for estimates of concentrations and the percentage of the population that was potentially infective. Thus, all oocysts were recovered without bias in estimates of $v_{0}$.

Determination of potential infectivity and quantification. Oocyst degradation was defined by several indicators of disruption of the oocyst wall and nuclei of sporozoites, including appearance at $\times 1,000$ magnification (obvious breaches in the oocyst wall), or the inclusion of propidium iodide (PI) within the oocyst (e.g., references 4 and 9).

We applied PI directly to filters, as previously described (9) (10 $\mu$ l of PI solution ( $1 \mathrm{~g}$ of PI:10 ml of $0.1 \mathrm{M}$ phosphate-buffered saline, $\mathrm{pH} 7.2$, with an incubation period of $90 \mathrm{~min}$ at $37^{\circ} \mathrm{C}$ ). This assay leads to conservative results with respect to degradation coefficients, because it overestimates the number of potentially infective oocysts present relative to other methods for demonstrating infectivity, e.g., the use of experimental animals (3). Following application of PI, we applied fluorescently labeled anti-Cryptosporidium immunoglobulin $\mathrm{M}$ according to the manufacturer's instructions (Waterborne, Inc.) and mounted filters on thick agar-coated slides beneath a coverslip with $20 \mu \mathrm{l}$ of fluorescence preservative gel mounting medium (gel and mount; Fisher Scientific, Pittsburgh, Pa.). We examined a minimum of 100 locations per slide.

Oocyst suspensions and exposures to temperature regimes. Oocysts were suspended in $\mathrm{NaCl}$ solutions by mixing $100 \mu \mathrm{l}$ of oocyst stock (containing $5 \times 10^{4}$ oocysts per $\mathrm{ml}$ ) with $1 \mathrm{ml}$ of $\mathrm{NaCl}$ solution in $1.5-\mathrm{ml}$ sealable polypropylene microfuge tubes. Triplicate suspensions were placed in aluminum heating blocks at $30^{\circ} \mathrm{C}$ or in plastic microcentrifuge tube racks in a refrigerator compartment at $4^{\circ} \mathrm{C}$ for 1,14 , and 29 days. For below-freezing temperatures $\left(-14^{\circ} \mathrm{C}\right)$, oocysts were suspended in $\mathrm{NaCl}$ solutions and placed in a freezer compartment in a plastic microcentrifuge tube rack for 1, 14, and 29 days. The tubes were in the central area of a frost-free freezer compartment, away from compartment walls and insulated from temperature fluctuations by stored materials. At the end of each time period, triplicates of each water potential condition were removed and thawed for $1 \mathrm{~h}$ at room temperature $\left(22 \pm 2^{\circ} \mathrm{C}\right)$.

For freeze-thaw cycling trials, oocysts were suspended in $\mathrm{NaCl}$ solutions and placed in the freezer compartment at $-14^{\circ} \mathrm{C}$. Freeze cycles lasted for $12 \mathrm{~h}$, prior to the entire batch being thawed in a refrigerated water bath at $10^{\circ} \mathrm{C}$ for $2 \mathrm{~h}$. We thawed tubes in a $10^{\circ} \mathrm{C}$ water bath to ensure that observed changes in oocyst characteristics represented freeze-thaw cycling rather than prolonged exposure to relatively high temperatures (e.g., room temperature). Triplicate sets were subjected to one to six cycles of freezing and thawing.

Data analysis. We represented population degradation with exponential decay models in a multiplicative error format (8). The dependent variable was the number of oocysts potentially capable of infecting a new host $\left(n_{3}\right)$ following exposure to stresses, relative to the starting number $\left(n_{1}\right)$ of oocysts capable of infecting a new host. Independent variables included fixed temperatures, exposure times, and levels of water potential.

Data analysis considered the following general model for outcomes of each experiment: $k=\vec{\beta} \vec{X}$, for $\vec{X}=\left(X_{1}, X_{2} \ldots X_{n}\right)$, in which $k=\ln \left(n_{3} / n_{1}\right) / t$ (the estimated degradation coefficient, day $\left.{ }^{-1}\right), t \equiv$ time of exposure (days) or number of freeze-thaw cycles, $n_{1} \equiv v_{0} \cdot n_{0}, n_{0} \equiv$ number of oocysts initially added to suspension, $v_{0} \equiv$ proportion of initial population of oocysts considered potentially infective $(0.92$ and 0.90 , for above- and below-freezing trials and freezethaw cycling trials, respectively), $n_{2} \equiv$ number of oocysts recovered from suspension following exposure to stresses, $v_{2} \equiv$ proportion of recovered population of oocysts considered potentially infective, $n_{3} \equiv v_{2} \cdot n_{2}, \vec{\beta} \equiv$ vector of coefficients estimated using linear regression $\left(\beta_{1}, \beta_{2} \ldots \beta_{n}\right)$ associated with levels of applied stresses, including duration of exposure and $\vec{X} \equiv$ vector of values representing levels of applied stresses.

The analyses applied regression through the origin to represent the assumption that the absence of temperature and water potential stresses would not affect degradation rates. The measure of multiple correlation often cited for linear regression $\left(r^{2}\right)$ is inappropriate for regression through the origin and is not presented to support the results of data analysis. Analysis of results tested experimental hypotheses by applying the significance of linear regression relationships (using $F$, with a rejection criterion of $P=0.05$ ) and significance of estimated coefficients (using $t$, with a rejection criterion of $P=0.05$ ). Individual and combined stresses were assumed significant if the regression relationship and coefficient estimates were significant at $P \leq 0.05$. All analyses were carried out with Minitab Release 12 (Minitab, Inc., State College, Pa.).

\section{RESULTS}

Suspensions at temperatures of $>0^{\circ} \mathrm{C}$. Water potential and temperature effects interact to increase the magnitude of the degradation coefficient relative to values expected from previous work (19). The regression model (Table 1) representing the relationship between the degradation coefficient at $4^{\circ}$ and $30^{\circ} \mathrm{C}$ and bar water potential was statistically significant at $P<$ 0.001 . Coefficient estimates for bars water potential and temperature were statistically significant at $P=0.003$ and $P<$ 


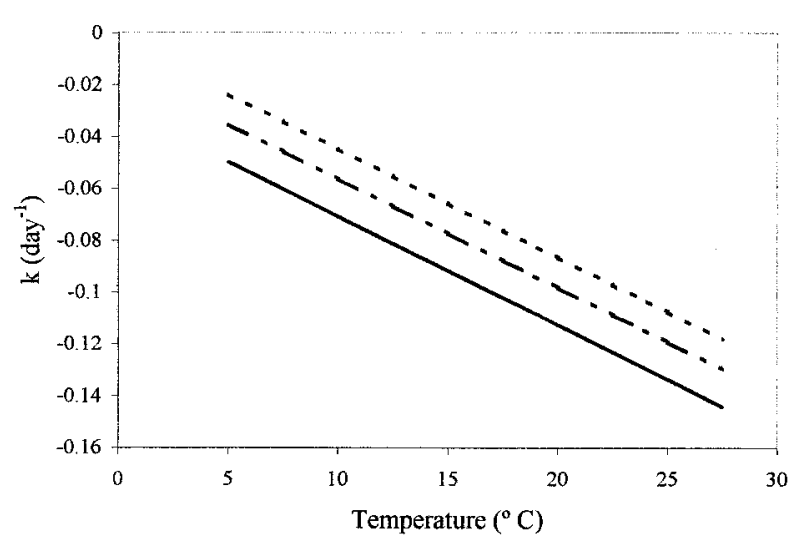

FIG. 1. Estimates of degradation coefficients $\left(k\right.$, day $\left.^{-1}\right)$ as a function of temperature (in degrees Celsius) and water potential ( -1 bar $[\cdots],-5$ bars [-- ], and -10 bars [-]), based on coefficient estimates reported for above-freezing conditions in Table 1.

0.001, respectively. These results indicate that, with increasing water potential stress, estimates of the degradation coefficient also increase (Fig. 1). As reported by other researchers (9), estimates of degradation coefficients increased in direct proportion to temperature increases. However, the results indicate that water potential stresses enhance the degradation rate, even under conditions in which temperature stresses are relatively minor.

Suspensions at $-\mathbf{1 4}^{\circ} \mathrm{C}$. A linear model of population decay (assuming the form of equation 1) considered the proportion of oocysts remaining as a variable dependent upon water potential at $-14^{\circ} \mathrm{C}$. The fitted model (Table 1) was significant at $P<0.001$, with the coefficient for bars water potential significant at $P=0.001$. The results indicate that water potential enhances the rates of reduction of oocyst numbers (Fig. 2).

Suspensions subjected to freeze-thaw cycling. The fitted model was significant at $P<0.001$, with the coefficient for bars water potential significant at $P<0.001$ (Table 1). The model suggests that water potential enhances the effects of freezethaw cycling (Fig. 2).

\section{DISCUSSION}

Relative effects of stresses. Water potential stresses enhance degradation due to above- and subfreezing temperature effects and freeze-thaw cycling. The values that we estimated are larger (on an absolute scale) than values previously used for risk assessment modeling related to public water supply protection (19) (Fig. 3). Among the stresses applied, freeze-thaw cycling is extreme and the effects of water potential are roughly four times those noted for freezing alone. The difference between effects of freeze-thaw cycling and simple freezing may be caused by mechanical damage to the oocyst wall (6). We expected such damage to increase as oocysts were subjected to more cycling events.

The results suggest that water potential conditions encountered under field conditions are likely to lead to more rapid degradation of oocyst populations than might be expected from studies of degradation in calf feces, distilled water with antibiotics, and reverse osmosis water at low temperatures (9)

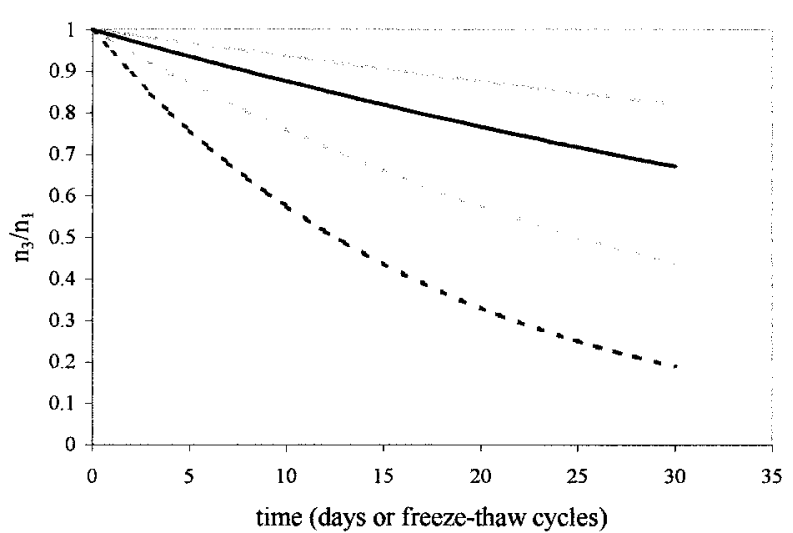

FIG. 2. Estimates of population degradation dynamics for exposure to subfreezing temperatures at water potential conditions of -0.5 bar (solid gray line) and -1.0 bar (solid black line) and freeze-thaw cycling conditions at water potential conditions of -0.5 bar (dotted gray line) and -1.0 bar (dotted black line). Graph depicts proportion of potentially infective oocysts remaining relative to the number added for each trial $\left(n_{3} / n_{1}\right)$ versus duration of exposure (days or number of freeze-thaw cycles). Note that estimates of degradation for freeze-thaw cycling are extrapolated beyond experimental conditions, which considered a maximum of six freeze-thaw cycles.

(Fig. 3). For example, at $4^{\circ} \mathrm{C}$ and -1 bar water potential, the estimated degradation coefficient is -0.046 day $^{-1}$, which is approximately an order of magnitude higher than previous estimates (9) that have been used to model the fate and transport of Cryptosporidium in public water supply watersheds (19).

Approaches to risk assessment modeling have relied on estimates of degradation coefficients that are conservative with respect to public health protection, although use of estimates derived from previous studies may be excessively conservative. Because water potential in the soil environment is likely to vary

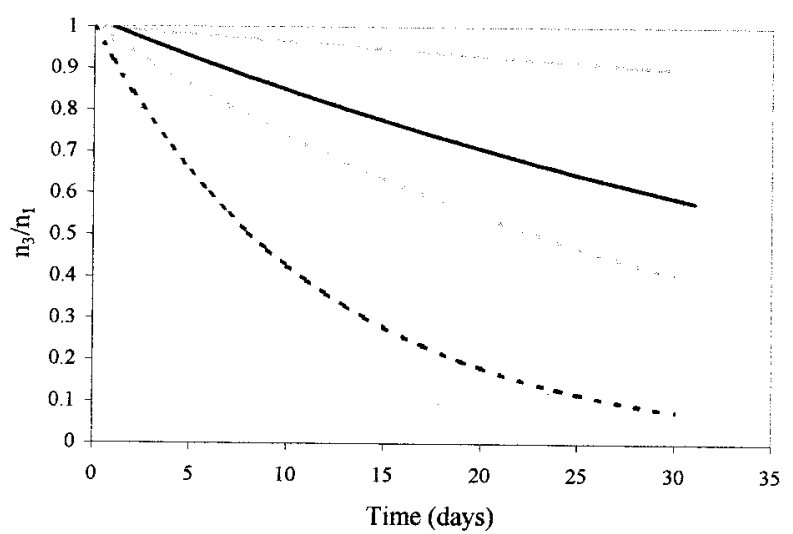

FIG. 3. Estimates of population degradation dynamics for abovefreezing conditions, based on temperature-dependent degradation coefficients (19) and temperature- and water potential-dependent degradation coefficients (reported for above-freezing temperatures [Table 1]). Estimates show differences between temperature-based estimates of the decay coefficient $k$ at 4 (solid gray line) and $20^{\circ} \mathrm{C}$ (dotted gray line) and temperature and water potential estimates at 4 (solid black line) and $20^{\circ} \mathrm{C}$ (dotted black line) over the course of 30 days with water potential stresses of -0.5 bars. Graph depicts proportion of potentially infective oocysts remaining relative to the number added for each trial $\left(n_{3} / n_{1}\right)$ versus duration of exposure (days). 
seasonally, with changes in soil moisture content, using a static degradation coefficient to characterize changes in oocyst populations may not represent the actual processes accurately. Water potential stresses in soils are predominantly exerted in combination as matric potential due to soil moisture content and osmotic potentials due to chemistry of soil solution (14). As an alternative, results of these experiments suggest that conservative approaches could be based on minimum degradation rates expected for water potential and temperature conditions at the soil surface, which may yield results that are conservative and are based on understanding of local conditions of expected soil temperature and water potential.

The multiplicative error model is used commonly to model the exponential growth and death phases of microbial population life cycles. This model assumes that stresses lead to changes in numbers that are best described by a single coefficient, $k$, which assumes negative values for the death phase (8) and represents a constant rate of reduction per time step. If the exponential models fitted above are accurate descriptions of population degradation, then those oocysts not destroyed by environmental stresses represent a subpopulation of concern. The results of these experiments and model fitting imply that small proportions of oocyst populations survive stresses. Because numbers of oocysts excreted by infected hosts may be extremely large (for example, $\sim 10^{10}$ over the course of illness in dairy calves [7], while the estimated infectious dose is $\sim 10^{2}$ [5]), small proportions of populations may represent very large numbers with respect to the potential to infect.

The implications of having small proportions of oocysts remaining infective following exposure to combined stresses is unclear. Prolonged exposure to natural stresses may render oocysts vulnerable to water disinfection processes. If so, the effects may enhance raw water disinfection processes. However, oocysts that survive stresses may be a resistant subpopulation that is more difficult to remove by disinfection processes commonly used for water treatment. In either case, experiments to evaluate performance of existing and new disinfection processes could consider using prestressed populations to evaluate treatment efficiency. Such experimental populations may best represent the treatment challenge faced by systems that potentially are influenced by nonpoint sources of oocysts.

\section{ACKNOWLEDGMENTS}

This research was supported in part by Hatch and USDA-NRI grants (Projects NEV0533F and NEV052HL, respectively).

We thank the reviewers for thoughtful and helpful comments.

\section{REFERENCES}

1. Adebayo, A., and R. Harris. 1971. Fungal growth responses to osmotic as compared to matric water potential. Soil Sci. Soc. Am. Proc. 35:465-469.
2. Brady, N. C. 1991. The nature and property of soils, 10th ed. Macmillan Publishing Co., New York, N.Y.

3. Bukhari, Z., M. Marshall, D. Korich, C. Fricker, H. Smith, J. Rosen, and J. Clancy. 2000. Comparison of Cryptosporidium parvum viability and infectivity assays following ozone treatment of oocysts. Appl. Environ. Microbiol. 66: 2972-2980.

4. Campbell, A. T., L. J. Robertson, and H. V. Smith. 1992. Viability of Cryptosporidium parvum oocysts: correlation of in vitro excystation with inclusion or exclusion of fluorogenic vital dyes. Appl. Environ. Microbiol. 58:34883493.

5. Dupont, H., C. Chappell, C. Sterling, P. Okhuysen, J. Rose, and W. Jakubowski. 1995 . The infectivity of Cryptosporidium parvum in healthy volunteers. N. Engl. J. Med. 332:855-859.

6. Fayer, R., T. Nerad, W. Rall, D. S. Lindsay, and B. L. Blagburn. 1991. Studies on cryopreservation of Cryptosporidium parvum. J. Parasitol. 77:357361.

7. Hoar, B. R., E. R. Atwill, T. B. Farver, and T. Jones. 2000. Estimating maximum possible environmental loading amounts of Cryptosporidium parvum attributable to adult beef cattle. Quant. Microbiol. 2:21-36.

8. Hurst, C. J., D. K. Wild, and R. M. Clark. 1992. Comparing the accuracy of equation formats for modeling microbial population decay rates, p. 149-175. In C. J. Hurst (ed.), Modeling the metabolic and physiologic activities of microorganisms. John Wiley \& Sons, New York, N.Y.

9. Jenkins, M. B., L. J. Anguish, M. J. Walker, D. D. Bowman, and W. C. Ghiorse. 1997. Assessment of a dye permeability assay for determination of inactivation rates of Cryptosporidium parvum oocysts. Appl. Environ. Microbiol. 63:3844-3850.

10. Kucznyska, E., and D. Shelton. 1999. Method for detection and enumeration of Cryptosporidium parvum oocysts in feces, manures, and soils. Appl. Environ. Microbiol. 65:2820-2826.

11. Laxer, M., B. Timblin, and R. Patel. 1991. DNA sequences for the specific detection of Cryptosporidium parvum by the polymerase chain reaction. Am. J. Trop. Med. Hyg. 199:688-694.

12. Mawdsley, J. L., A. E. Brooks, and R. J. Merry. 1996. Movement of the protozoan pathogen Cryptosporidium parvum through three contrasting soil types. Biol. Fertil. Soils 21:30-36.

13. Moore, J. A. 1991. Surface transport of microorganisms by water, p. 41-52. In L. R. Ginzburg (ed.), Assessing ecological risks of bio/technology. Butterworth-Heinemann, Boston, Mass.

14. Papendick, R. I., and G. S. Campbell. 1978. Theory and measurement of water potential, p. 1-21. In D. Kral (ed.), Water potential relations in soil microbiology. SSA special publication no. 9. Soil Science Society of America, Madison, Wis.

15. Rawlins, S., and G. Campbell. 1986. Water potential: thermocouple psychrometry, p. 597-616. In A. L. Page, R. H. Miller, and D. R. Keeney (ed.), Methods of soil analysis, part 1. Physical and mineralogical methods. Agronomy monograph no. 9, 2nd ed. American Society of Agronomy-Soil Science Society of America, Madison, Wis.

16. Robertson, L., A. Campbell, and H. Smith. 1992. Survival of Cryptosporidium parvum oocysts under various environmental pressures. Appl. Environ. Microbiol. 58:3494-3500.

17. U.S. Environmental Protection Agency. 1999. Method 1622: Cryptosporidium in water by filtration. IMS/FA EPA-821-R-99-001. Engineering and Analysis Division, Office of Water, U.S. Environmental Protection Agency, Washington, D.C.

18. Vetterling, J. 1969. Continuous-flow differential density flotation of coccidial oocysts and a comparison with other methods. J. Parasitol. 55:412-417.

19. Walker, F. R., and J. R. Stedinger. 1999. Fate and transport model of Cryptosporidium. J. Environ. Eng. 125:325-333.

20. Walker, M., C. Montemagno, and M. Jenkins. 1998. Source water assessment and nonpoint sources of acutely toxic contaminants: a review of research related to survival and transport of Cryptosporidium parvum. Water Resour. Res. 34:3383-3392. 